\author{
Р.В. Казымлы ${ }^{1}$, Р.О. Гусейнова ${ }^{2}$ \\ ${ }^{1}$ Национальное аэрокосмическое агентство, Баку, \\ Азербайджанская Республика \\ ${ }^{2}$ Азербайджанский университет архитектуры и строительства, \\ Баку, Азербайджанская Республика

\section{ВОПРОСЫ ОБЕСПЕЧЕНИЯ ЭФФЕКТИВНОСТИ ЛАЗЕРНОЙ СВЯЗИ МЕЖДУ УЗЛАМИ РАСПРЕДЕЛЕННЫХ ИЗМЕРИТЕЛЬНЫХ СЕТЕЙ}

\begin{abstract}
Рассматриваются вопросы обеспечения эффективности лазерной связи между узлами распределенных измерительных сетей. Одним из основных воздействующих факторов, приводящих к ослаблению сигнала диодных лазеров, является дивергенция (расхождение) луча, которая может достичь 3-8 mrad. Также важным фрактором является турбулентность атмосферы, которая возникает из-за разности температур поверхности Земли и атмосферы. В атмосферных лазерных линиях связи основными воздействующими внешними фракторами являются туман, снег, дождь. С учетом воздействия указанных факторов предложена новая модель ослабления лазерного сигнала в открытой коммуникационной сети между центральным узлом и распределенными приемными узлами. В предложенной модели в качестве основных рассматриваются такие показатели, как расстояние между центром и распределенными узлами; видимость на дистанции и показатель распределения рассеивающих частиц. Предложен новый показатель эффективности коммуникации в распределенных лазерных сетях. Составлена и решена задача нахождения экстремума предложенного показателя. На основе полученного решения оптимизационной задачи даны рекомендации по выбору взаимосвязи длины дистанции и видимости на этой дистанции. Заключено, что в лазерной сети коммуникации между центральным узлом и распределенными узлами связи наихудший по выбранному обобщенному показателю эффективности результат может быть получен при возрастающем характере функциональной связи между расстоянием и видимостью, т.е. с ростом видимости при увеличении расстояния до выбранного приемного узла. Эвристической альтернативой в данном случае является организация обратной зависимости между расстоянием и видимостью, т.е. такой вариант, когда уменьшение расстояния сопровождалось бы увеличением видимости.
\end{abstract}

Ключевые слова: лазерная связь, эффективность, оптимизация, измерительные сети, видимость. 


\author{
R.V. Kazimli ${ }^{1}$, R.O. Huseynova ${ }^{2}$ \\ ${ }^{1}$ National Aerospace Agency, Baku, Azerbaijan Republic \\ ${ }^{2}$ Azerbaijan University of Architecture and Construction, \\ Baku, Azerbaijan Republic
}

\title{
QUESTIONS ON PROVIDING EFFECTIVENESS OF LASER COMMUNICATION BETWEEN NODES OF DISTRIBUTED MEASUREMENTS NETWORKS
}

The article is devoted to questions on providing effectiveness of laser communication between nodes of distributed measuring networks. One of major impacting factors leading to attenuation diode laser signals is divergence of beam which can reach 3-8 mrad. Another important factor is atmospheric turbulence which occurs due to difference of temperatures of the Earth surface and atmosphere. In atmospheric laser communication lines major impacting factors are mist, snow and rain. In view of said factors the new model of attenuation of laser signal in open communication network between the central node and distributed receiving nodes is suggested. In suggested model such parameters as distance between center and distributed nodes, visibility at the distance and scattering particles distribution parameter are considered as major ones. The new indicator of effectiveness of communication in distributed laser networks is suggested. The task on analysis of suggested indicator is formulated and solved. On the basis of solution of optimization task the recommendations on choosing interrelation between length of distance and visibility at the distance are given. It is concluded that in laser communication network the worst effectiveness on chosen relevant parameter between central node and distributed ones can be obtained upon increasing type of functional dependence between distance and visibility i.e. when increase of visibility is caused by increase of distance till chosen receiving node. Heuristic alternative in this case is organization of inverse dependence between distance and visibility, i.e. when decrease of distance is accompanied by increase of visibility.

Keywords: laser communication, effectiveness, optimization, measuring networks, visibility.

Введение. Как отмечается в [1-7], изменения мощности приемного сигнала в беспроводных измерительных сетях могут быть вызваны эффектами потерь из-за диссипации энергии сигнала передатчика на дистанции, а также явлением «затенения», вызванным такими событиями, как поглощение, отражение, рассеяние и дифракция.

Существует множество моделей распространения радиосигналов, которые разделяются на модели распространения в пределах помещения и вне помещения [8-12]. Эти модели, охватывая частотный диапазон 40 мГц - 100 гГц представляют собой относительно несложные математические выражения, связывающие внешние воздействующие факторы и уровень ослабления переданного сигнала на приемнике.

Вместе с тем, если перейти на лазерные линии коммуникации, то здесь не обнаруживается большого множества моделей передачи и распространения сигнала. Согласно [13] одним из основных воздей- 
ствующих факторов, приводящих к ослаблению сигнала диодных лазеров является дивергенция (расхождение) луча, которое может достичь 3-8 mrad. Не менее важным фактором является турбулентность атмосферы, которая возникает из-за разности температур поверхности Земли и атмосферы. В атмосферных лазерных линиях связи основными воздействующими внешними факторами являются туман, снег, дождь. Воздействие этих факторов отображено на рис. 1 в виде зависимости атмосферного ослабления от видимости [14-20].

Согласно [14] имеется техническое определение видимости, по которому видимость - это такое расстояние, где оптический сигнал уменьшается по мощности до $2 \%$ исходной мощности. Согласно качественному определению видимости это такое расстояние, когда все еще возможно различить темный объект на горизонте.

Вместе с тем при проектировании открытых линий оптических коммуникаций одним из основных показателей является требуемое расстояние между передатчиком и приемником, которое и определяет необходимый набор мер обеспечения надежной связи на рассматриваемой дистанции. Далее в настоящей статье мы рассмотрим ту потенциальную взаимосвязь между модельными показателями лазерной линии коммуникации, при которой достигаются экстремальные значения вновь вводимых показателей коммуникационных лазерных сетей.

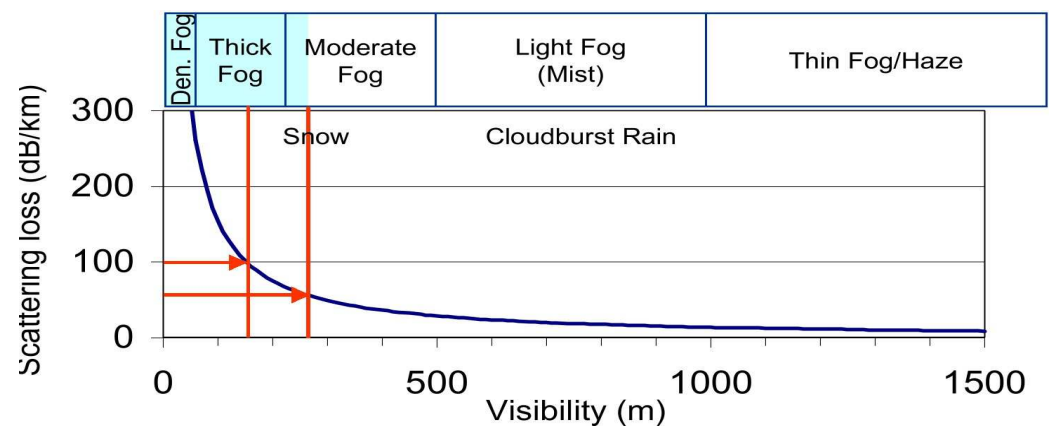

Рис. 1. Роль факторов зависимости атмосферного ослабления от видимости [14]

\section{1. Предлагаемая модель лазерной коммуникационной сети.}

Далее рассматривается модель передачи лазерных сигналов от центрального узла к периферийным узлам, находящимся на различных расстояниях от центрального узла, а также отличающихся специфическими условиями распространения лазерного луча (рис. 2). 


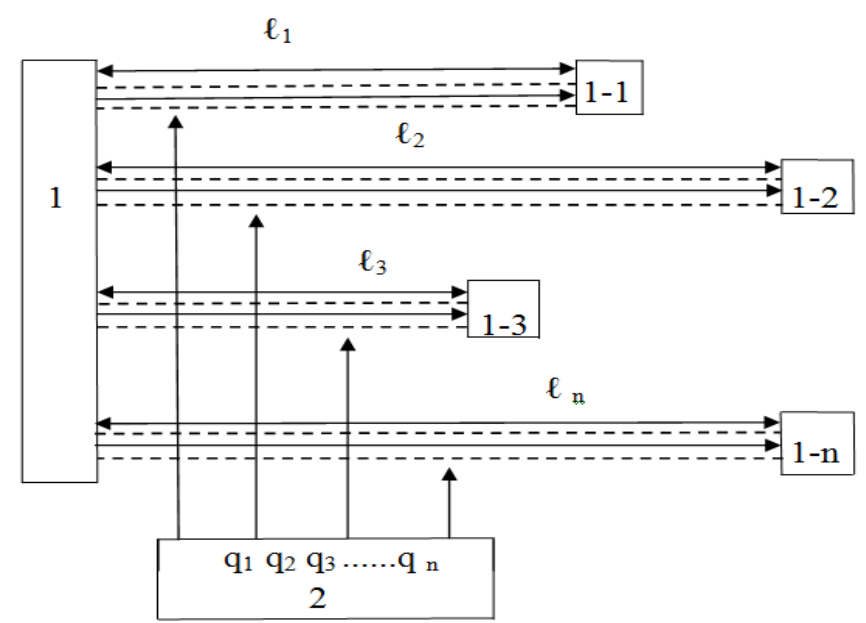

Рис. 2. Предлагаемая модель распространения лазерного луча в открытых лазерных коммуникационных сетях распределенных узлов связи

На рис. 2 цифрами показаны: 1 - центральный узел передачи; $(1-i)$; $i=\overline{(1, n)}$ - приемные узлы; 2 - узел, моделирующий воздействие внешних факторов через показатель $q_{i}$, определяющий видимость на трассе.

Согласно [9] ослабление мощности сигнала в атмосфере определяется известным законом Бугера-Бера-Ламберта:

$$
P(R)=P(0) \cdot e^{-G R},
$$

где $P(R)$ - мощность лазерного сигнала на дистанции $R ; P(0)$ - мощность лазерного сигнала на источнике; $G$ - ослабление или суммарная экстинкция оптического сигнала на единичном пути.

При этом пропускание атмосферы на дистанции $R$ определяется как

$$
\tau(R)=\frac{P(R)}{P(0)}=e^{-G R} .
$$

Согласно [9], поскольку эффективность рассеяния лазерного луча является функцией как параметра атмосферных частиц $r$, так и отношения $r / \lambda$, где $r$ - радиус атмосферных частиц; $\lambda$ - длина волны, то имеет место соотношение

$$
G=A \cdot \lambda^{-q},
$$

где $A$ и $q$ - показатели, определяемые размером и распределением рассеивающих частиц. 
Существует [9] следующее известное соотношение (4), связывающее следующие показатели: $G$ - коэффициент атмосферного ослабления (рассеяния); $V$ - видимость (в км); $\lambda$ - длина волны (нм); $q$ - показатель распределения рассеивающих частиц, определяемый в зависимости от $V$ следующим образом (таблица):

\begin{tabular}{|c|c|c|c|}
\hline$V$ & $V<6$ км & 6 км $<V<50$ км & $V>50$ км \\
\hline$q$ & $0,585 \cdot V^{\frac{1}{3}}$ & 1,3 & 1,6 \\
\hline
\end{tabular}

$$
G=\frac{3,91}{V}\left(\frac{\lambda}{550 \mathrm{HM}}\right)^{-q} .
$$

С учетом выражений (1), (3), (4) имеем:

$$
P(R, q)=P(0) \exp \left[-\frac{3,91}{V}\left(\frac{\lambda}{550 \mathrm{HM}}\right)^{-q} \cdot R\right] .
$$

Как видно из предложенной модели (см. рис. 2), в коммуникационной сети объективно существуют два множества независимых параметров:

$$
\begin{aligned}
& R=\left\{R_{i}\right\}, i=\overline{(1, n)}, \\
& Q=\left\{q_{j}\right\}, j=\overline{(1, n)} .
\end{aligned}
$$

3. Составление оптимизационной задачи. Далее решается оптимизационная задача нахождения такой взаимосвязи $R_{i}$ и $q_{j}$, $(i, j=\overline{1, n)}, R=f(q)$, при которой нижеуказанный предлагаемый обобщенный показатель сети достиг бы экстремальной величины:

$$
\int_{q_{\min }}^{q_{\max }} P(R, q) d q=\int_{q_{\min }}^{q_{\max }} P(0) \exp \left[-\frac{3,91}{V}\left(\frac{\lambda}{550 \mathrm{HM}}\right)^{-q} \cdot K(q)\right] d q .
$$

Заметим, что целевой функционал (8) эффективности является идеализированным случаем перехода от следующей дискретной модели к непрерывной модели:

$$
\sum_{q_{i}} P\left(R_{i}, q_{i}\right)=\sum_{q_{i}} P(0) \exp \left[-\frac{3,91}{V}\left(\frac{\lambda}{550 \mathrm{HM}}\right)^{-q} \cdot\left(R_{i} q_{j}\right)\right] .
$$


Для решения задачи вычисления оптимальной функции $R(q)$ несколько сузим класс рассматриваемых возможных функций и предположим, что реально рассматриваемые функции $R(q)$ должны выполнить следующее ограничительное условие:

$$
\int_{q_{\min }}^{q_{\max }} R(q) d q=C, C=\text { const. }
$$

С учетом выражений (8) и (10) составим заключительный показатель эффективности - функционал безусловной вариационной оптимизации, условно приняв $q_{\min }=0$ :

$$
\int_{0}^{q_{\max }} P(0) \exp \left[-\frac{3,91}{V}\left(\frac{\lambda}{550 \mathrm{HM}}\right)^{-q} \cdot R(q)\right] d q+\gamma\left[\int_{0}^{q_{\max }} R(q) d q-C\right],
$$

где $\gamma$ - множитель Лагранжа.

4. Решение оптимизационной задачи. Решение $R(q)_{\text {опт }}$, приводящее $F$ к его экстремуму, вычисляется согласно уравнению ЭйлераЛагранжа по условию:

$$
\frac{d\left\{P(0) \exp \left[-\frac{3,91}{V}\left(\frac{\lambda}{550 \mathrm{Hм}}\right)^{-q} \cdot R(q)\right]+\gamma[R(q) d q-C]\right\}}{d R(q)}=0 .
$$

Решение оптимизационной задачи с учетом (12) получено в виде

$$
R(q)=\frac{1}{a_{1}} \cdot \ln \left[\frac{P\left(0 \cdot a_{1}\right)}{\gamma_{0}}\right]
$$

где

$$
\begin{gathered}
\gamma_{0}=\exp \left[\frac{\int_{0}^{q_{\max }} 1 / a_{1} \ln \left[P(0) \cdot a_{1}\right] d q-C}{\int_{0}^{q_{\max }} d q / a_{1}}\right] . \\
a_{1}=\frac{3,91}{V}\left(\frac{\lambda}{550 \mathrm{HM}}\right)^{-q} .
\end{gathered}
$$

При этом показано, что при решении (13)-(15) целевой функционал (11) достигает минимальной величины. 
Таким образом, с учетом решенной оптимизационной задачи можно рассмотреть трехмерное пространство $\{R, q, V\}$, где зависимость $q=f_{1}(V)$ задана по данным таблицы; $R=f_{2}(q)$ - определяется по вычисленному выражению (13), а зависимость $R=f_{3}(V)$ вычисляется путем геометрического построения (рис. 3).

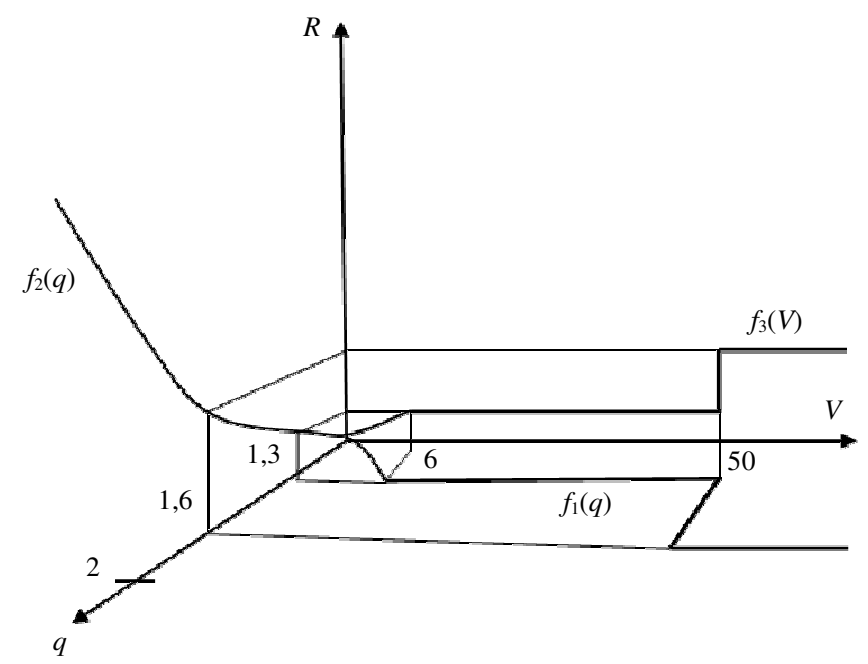

Рис. 3. Геометрическое построение функции $R=f_{3}(V)$, при которой целевой функционал (11) достигает минимума

Выводы. Таким образом, можно заключить, что в лазерной сети коммуникации между центральным узлом и распределенными узлами связи наихудший по выбранному обобщенному показателю эффективности результат может быть получен при возрастающем характере функции $R=f_{3}(V)$, т.е. с ростом видимости при увеличении дистанции $R$ до выбранного приемного узла.

Очевидно, что эвристической альтернативой в данном случае является организация обратной зависимости между $R$ и $V$, т.е. такой вариант, когда уменьшение $R$ сопровождался бы увеличением видимости.

Сформулируем основные выводы проведенного исследования:

1. Предложения новая модель ослабления лазерного сигнала в открытой коммуникационной сети между центральным узлом и распределенными приемными узлами, в которой в качестве основных рассматриваются такие показатели, как расстояние между центром и распределенными узлами; видимость на дистанции и показатель распределения рассеивающих частиц. 
2. Предложен новый показатель эффективности коммуникации в распределенных лазерных сетях. Составлена и решена задача нахождения экстремума предложенного показателя.

3. На основе полученного решения оптимизационной задачи даны рекомендации по выбору взаимосвязи длины дистанции и видимости на этой дистанции.

\section{Библиографический список}

1. Naseem Z., Nausheen I., Mirza Z. Propagation models for wireless communication system // International Research Journal of Engineering and Technology. - January 2018. - Vol. 05, iss. 01. - P. 237-242.

2. Kim Isaac I., McArthur Bruce, Korevaar Eric. Comparison of laser beam propagation at $785 \mathrm{~nm}$ and $1550 \mathrm{~nm}$ in fog and haze for optical wireless communications ERDG/CERL, TR-06-04 . - URL: https://spie.org/ Publications/Proceedings/Paper/10.1117/12.417512?origin_id=x4325\&start _volume_number $=04200$

3. Dordova Lucie, Wilfelt Otakar. Laserbeam attenuation determined by the method of available optical power in turbulent atmosphere // Journal of telecommunications and information technology. - 2009. - 2. - P. 53-58.

4. Naseem Zhera, Nausheen Iram, Mirza Zahwa. Propagation models for wireless communication system // International Research Journal of Energineering and Technology. - 01.01.2018. - Vol. 05. - P. 237-341.

5. Localization, tracking, and imaging of targets in wireless sensor networks / F. Viani, P. Rocca, G. Oliveri, D. Trinchero, A. Massa // An invited review. - URL: http://citeseerx.ist.psu.edu/viewdoc/download?doi= 10.1.1.473.8534\&rep=rep1\&type $=$ pdf

6. Perez-Vega Costantino, Garcia G. Jose Luis. Frequency behavior of a power-law path loss model. - URL: https://www.researchgate.net/publication/ 239924028_Frequency_behavior_of_a_power-law_path_loss_model

7. Atmospheric influences on satellite communications / Zubair Muhammad, Haider Zaffar, Akhan Shahid, Nasir Jamob / Comsats Institute of Information Technology, Islamabad (1,2,3); Comsats Institute of Information Technology Abbott Abad (4) // Przeglad elektrotechniczny (Electrical Review). - 2011. - Vol. 87. - № 5.

8. Calla O.P.N., Purohit J.S. Study of effect of rain and dust on propagation of radio waves at millimeter wavelength. - URL: http://citeseerx.ist.psu. edu/viewdoc/download?doi=10.1.1.491.9393\&rep=rep1\&type=pdf 
9. Dordova L., Wilfert O. Optimal laser diode operating mode with unstable operating temperature in turbulent atmosphere // Proc. Semicond. Laser Dynam. SPIE Eur. Photon. Eur. 2008. - Strasbourg, France, 2008. - P. 1-11.

10. Tosovsky O., Dordova L. Free space optical channel parameters estimation for high altitude platform system // Proc. 18 ${ }^{\text {th }}$ Int. Conf. Radioelektr. - Prague, Czech Republic. - 2008. - P. 1-5.

11. Koreevar E., Kim I., MacArthur B. Atmospheric propagation characteristic of highest importace to commercial free space optics // Proc. SPIE (Atmospheric propagation). - 2003. - Vol. 4976. - P. 1-12.

12. Naboulsi A., Sizun M., Fornel F.De. Propagation of optical and infrared waves in the atmosphere // Proc. $28^{\text {th }}$ URSI Gener. Assem. - New Dehli, India, 2005. - P. 1-4.

13. Dordova L., Wilfert O. Laser beam attenuation determined the method of available optical power in turbulent atmosphere // Journal of Telecommunications and Information technology. - 2009. - № 2. - P. 53-57.

14. Kim I.I., MacArthur B., Koreevar E. Comparison of laser beam propagation at 785 and $1550 \mathrm{~nm}$ in fog and haze for optical wireless communication//ERDC/CERL. - TR-06-4. - P. 21.

15. Measurement of scintillation and link margin for the TerraLink laser communication system / I.I. Kim, J. Koontz, H. Hakakha, P. Adhikari, R. Stieger, C. Moursund, M. Barclay, A. Stanford, R. Ruigrok, J. Schuster, E. Koreevar // Wireless Technology System: Mimmimeter Wave and Optical. Proc. SPIE. - 1997. - Vol. 3232. - P. 100-118.

16. Wireless optical transmission of Fast Ethernet, FDDI, ATM and Escon protocol data using the TerraLink laser communication system / I.I. Kim, R. Stieger, J. Koontz, C. Moursund, M. Barclay, P. Adhikari, J. Schuster, E. Koreevar // Opt. Eng. - 1998. -Vol. 37. - P. 3143-3155.

17. $2.4 \mathrm{rv}$ free-space optical communication $1550 \mathrm{~nm}$ transmission link operating at $2.5 \mathrm{~Gb} / \mathrm{s}$ - experimental results / P.F. Szajowski, G. Nykolak, J.J. Auborn, H.M. Presby, J.A. Abate, G.E. Tourgee, E. Koreevar, J. Schuster, I.I. Kim // Optical Wireless Communication. Proc. SPIE. - 1998. Vol. 3532. - P. 29-40.

18. $4 \times 2.5 \mathrm{~Gb} / \mathrm{s} 4.4 \mathrm{~km}$ WDM free-space optical link at $1550 \mathrm{~nm} /$ G. Nykolak, P.F. Szajowski, J. Jacques, H.M. Presby, J.A. Abate, G.E. Tourgee, J.J. Auborn // Post Deadline Paper Optical Fiber Conference. - 1999. 
19. High power optical amplifier enable $1550 \mathrm{~nm}$ terrestrial free-space optical dala-link operating @WDM $2.5 \mathrm{~Gb} / \mathrm{s}$ data rates / P.F. Szajowski, J.J. Auborn, G. Nykolak, H.M. Presby, G.E. Tourgee // Optical Wireless Communication II Proc. SPIE. - 1999. - Vol. 3850. - P. 2-10.

20. Update on $4 \times 2.5 \mathrm{~Gb} / \mathrm{s} 4.4 \mathrm{~km}$ free-space optical communication link availability and scintillation performance / G. Nykolak, J.J. Auborn, H.M. Presby, P.F. Szajowski, G.E. Tourgee // Optical Wireless Communication II Proc. SPIE. - 1999. - Vol. 3850. - P. 11-19.

\section{References}

1. Naseem Z., Nausheen I., Mirza Z. Propagation models for wireless communication system. International Research Journal of Engineering and Technology. January, 2018, vol. 05, iss. 01, pp. 237-242.

2. Kim Isaac I., McArthur Bruce, Korevaar Eric. Comparison of laser beam propagation at $785 \mathrm{~nm}$ and $1550 \mathrm{~nm}$ in fog and haze for optical wireless communications ERDG/CERL, TR-06-04, available at: https://spie.org/Publications/Proceedings/Paper/10.1117/12.417512?origin_i $\mathrm{d}=\mathrm{x}$ 4325\&start_volume_number $=04200$

3. Dordova Lucie, Wilfelt Otakar. Laserbeam attenuation determined by the method of available optical power in turbulent atmosphere. Journal of telecommunications and information technology, 2009, 2, pp. 53-58.

4. Naseem Zhera, Nausheen Iram, Mirza Zahwa. Propagation models for wireless communication system. International Research Journal of Energineering and Technology, 01.01.2018, vol. 05, pp. 237-341.

5. Viani F., Rocca P., Oliveri G., Trinchero D., Massa A. Localization, tracking, and imaging of targets in wireless sensor networks: An invited review, available at: http://citeseerx.ist.psu.edu/viewdoc/ download?doi=10.1.1.473.8534\&rep=rep1\&type=pdf

6. Perez-Vega Costantino, Garcia G. Jose Luis. Frequency behavior of a power-law path loss model, available at: https://www.researchgate.net/publication/239924028_Frequency_behavior_of_a_power-law_path_loss_model

7. Zubair Muhammad, Haider Zaffar, Akhan Shahid, Nasir Jamob. Atmospheric influences on satellite communications. Comsats Institute of Information Technology, Islamabad $(1,2,3)$. Comsats Institute of Information Technology Abbott Abad (4). Przeglad elektrotechniczny (Electrical Review), 2011, vol. 87, no. 5. 
8. Calla O.P.N., Purohit J.S. Study of effect of rain and dust on propagation of radio waves at millimeter wavelength, available at: http://citeseerx.ist.psu.edu/viewdoc/download?doi=10.1.1.491.9393\&rep=re $\mathrm{p} 1 \&$ type $=\mathrm{pdf}$

9. Dordova L., Wilfert O. Optimal laser diode operating mode with unstable operating temperature in turbulent atmosphere. Proc. Semicond. Laser Dynam. SPIE Eur. Photon. Eur. 2008. Strasbourg, France, 2008, pp. 1-11.

10. Tosovsky O., Dordova L. Free space optical channel parameters estimation for high altitude platform system. Proc. 18 ${ }^{\text {th }}$ Int. Conf. Radioelektr. Prague, Czech Republic, 2008, pp. 1-5.

11. Koreevar E., Kim I., MacArthur B. Atmospheric propagation characteristic of highest importace to commercial free space optics. Proc. SPIE (Atmospheric propagation), 2003, vol. 4976, pp. 1-12.

12. Naboulsi A., Sizun M., Fornel F.De. Propagation of optical and infrared waves in the atmosphere. Proc. $28^{\text {th }}$ URSI Gener. Assem. New Dehli, India, 2005, pp. 1-4.

13. Dordova L., Wilfert O. Laser beam attenuation determined the method of available optical power in turbulent atmosphere. Journal of Telecommunications and Information technology, 2009, no. 2, pp. 53-57.

14. Kim I.I., MacArthur B., Koreevar E. Comparison of laser beam propagation at 785 and $1550 \mathrm{~nm}$ in fog and haze for optical wireless communication//ERDC/CERL, TR-06-4, P. 21.

15. Kim I.I., Koontz J., Hakakha H., Adhikari P., Stieger R., Moursund C., Barclay M., Stanford A., Ruigrok R., Schuster J., Koreevar E. Measurement of scintillation and link margin for the TerraLink laser communication system. Wireless Technology System: Mimmimeter Wave and Optical. Proc. SPIE, 1997, vol. 3232, pp. 100-118.

16. Kim I.I., Stieger R., Koontz J., Moursund C., Barclay M., Adhikari P., Schuster J., Koreevar E. Wireless optical transmission of Fast Ethernet, FDDI, ATM and Escon protocol data using the TerraLink laser communication system. Opt. Eng, 1998, vol. 37, pp. 3143-3155.

17. Szajowski P.F., Nykolak G., Auborn J.J., Presby H.M., Abate J.A., Tourgee G.E., Koreevar E., Schuster J., Kim I.I. 2.4 rv freespace optical communication $1550 \mathrm{~nm}$ transmission link operating at 
$2.5 \mathrm{~Gb} / \mathrm{s}$ - experimental results. Optical Wireless Communication. Proc. SPIE, 1998, vol. 3532, pp. 29-40.

18. Nykolak G., Szajowski P.F., Jacques J., Presby H.M. Abate, J.A., Tourgee G.E., Auborn J.J. $4 \times 2.5 \mathrm{~Gb} / \mathrm{s} 4.4 \mathrm{~km}$ WDM free-space optical link at 1550 nm. Post Deadline Paper Optical Fiber Conference, 1999.

19. Szajowski P.F., Auborn J.J., Nykolak G., Presby H.M., Tourgee G.E. High power optical amplifier enable $1550 \mathrm{~nm}$ terrestrial free-space optical dala-link operating @WDM $2.5 \mathrm{~Gb} / \mathrm{s}$ data rates. Optical Wireless Communication II Proc. SPIE, 1999, vol. 3850, pp. 2-10.

20. Nykolak G., Auborn J.J., Presby H.M., Szajowski P.F., Tourgee G.E. Update on $4 \times 2.5 \mathrm{~Gb} / \mathrm{s} 4.4 \mathrm{~km}$ free-space optical communication link availability and scintillation performance. Optical Wireless Communication II Proc. SPIE, 1999, vol. 3850, pp. 11-19.

\section{Сведения об авторах}

Казымлы Рейхана Вагиф гызы (Баку, Азербайджанская Республика) - докторант (аспирант) Национального аэрокосмического агентства (AZ1115, Баку, ул. С.С. Ахундова, 1, e-mail: reyhana.kazimli@gmail.com).

Гусейнова Рена Омар гызы (Баку, Азербайджанская Республика) - доктор философии в области техники, доцент Азербайджанского архитектурно-строительного университета (AZ 1073, Баку, ул. Айны Султановой, 5, e-mail: renahuseynova55@gmail.com).

\section{About the authors}

Kazimli Reyhane Vaqif qizi (Baku, Azerbaijan Republic) is a Doctorate National Aerospace Agency (AZ1115, Baku, 1, S.S. Akhundov str., e-mail: reyhana.kazimli@gmail.com).

Huseynova Rena Omar qizi (Baku, Azerbaijan Republic) is a Ph.D in Technical Science, Assistant Professor of Azerbaijan University of Architecture and Construction (AZ1073, Baku, 5, Aina Sultanova str., e-mail: renahuseynova55@gmail.com).

Получено 30.10.2019 